DOE/JPL

Distribution Category UC-63

Report \#5

\title{
DEFECT STRUCTURE OF WEB SILICON RIBBON
}

October 1980

JPL Contract No. 954852

MASTER

by

B. Cunningham, H. Strunk and D. Ast

\author{
Materials Science and Engineering \\ Bard Hall, Cornell University \\ Ithaca, New York 14853
}

The JPL low-cost solar array project is sponsored by the U.S. Department of Energy and forms part of the Solar Photovoltaic Conversion Program to initiate a major effort toward the development of low-cost solar arrays. This work was performed for the Jet Propulsion Laboratory, California Institute of Technology by agreement between NASA and DOE.

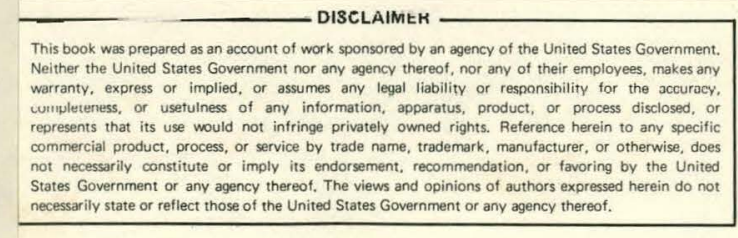




\section{DISCLAIMER}

This report was prepared as an account of work sponsored by an agency of the United States Government. Neither the United States Government nor any agency Thereof, nor any of their employees, makes any warranty, express or implied, or assumes any legal liability or responsibility for the accuracy, completeness, or usefulness of any information, apparatus, product, or process disclosed, or represents that its use would not infringe privately owned rights. Reference herein to any specific commercial product, process, or service by trade name, trademark, manufacturer, or otherwise does not necessarily constitute or imply its endorsement, recommendation, or favoring by the United States Government or any agency thereof. The views and opinions of authors expressed herein do not necessarily state or reflect those of the United States Government or any agency thereof. 


\section{DISCLAIMER}

Portions of this document may be illegible in electronic image products. Images are produced from the best available original document. 


\section{ABSTRACT}

Web silicon ribbon has recently emerged as a material for the production of high efficiency. solar cells. Since defects introduced during growth may influence locally minority carrier recombination rates, there is now a need to examine the defect structure in detail and to correlate it with "electrical activity". This work describes initial observations made on web material by EBIC and HVEM.

Although EBIC investigations have shown that dislocations emerging at the web surface enhance minority carrier recombination rates, their density is low enough (typicaliy $10^{5} \mathrm{~cm}^{-2}$ ) to have oniy a small effect on the efficiency of the material as a solar cell. Since a condition for dendritic web growth is that the dendrites contain at least two twin boundaries it is usual to find that some of these boundaries extend into the web. These boundaries are formed parallel to the (III) growth surface and are found to be sites of strong electrical activity.

HVEM has been used to study the defect structure at the twin boundary. Two types of dislocation networks lying on different $\{111\}$ planes have been observed, presumably corresponding to two adjacent twin boundaries: One network consists of an hexagonal array of twin boundary partial dislocations interacting with Lomer-Cottrell locks. The other network is made up of both perfect and partial dislocations running in the [2Īi] growth direction. 


\section{Introduction}

The growth of silicon ribbons by the dendritic web process was first discussed by Dermatis and Faust(1) and O'Hara and Bennett(2) in the early 1960's. The current interest in silicon web is due to its potential use as a șibstrate

material for the economic production of high efficiency solar cells. Seidensticker(3) has shown that the characteristics of solar cells fabricated from dendritic web are virtually identical to those made from the more commonly grown Czochralski material.

Since crystalline defects in semiconductor materials frequently affect the electrical properties, e.g. minority carrier lifetime, the structure and electrical activity of the defects in silicon web. were the subjects of the present investigation.

This report summarizes the results of a preliminary study of two Westinghouse dendritic web samples, identification numbers WI80-3.4C and JI35-3.6.

\section{Experimental Techniques}

Optical microscopy of chemically etched specimens was used to determine dislocation densities. Samples were mechanically polished, then Secco(4) etched for approximately 5 mins.

High voltage transmission electron microscopy (HVTEM) was used to characterize the crystallographic nature of the defects. The twin planes in web material are parallel to the surface (and at or near the center of the ribbon). The web thickness is only $\sim 150 \mu m$ and, for this reason, it is difficult to prepare a specimen for TEM where the twin boundaries can be viewed end-on. Specimens for the present investigation were therefore thinned as symetrically as possible from both surfaces. WIth HIV ${ }_{3}$ :HF:HAc $(5: 1: 1)$ until a hole with tapered edges was formed. The centrally located twin boundaries were, however, generally not contained in the very thin $(\alpha \varkappa 0.5 \mu \mathrm{m})$ annulus around the hole that can be inspected by 
conventional (100 keV) transmission electron microscopy. The use of a high voltage microscope ( $1 \mathrm{MeV}$ ) with its large penetrating power of several $\mu \mathrm{m}$ proved to be essential to image the twin plane or planes at the approximate center of the ribbon.

The electronic properties of the material were studied using a scanning electron microscope (SEM) operating in the EBIC mode (electron beam induced current). In this mode the electron beam is used to inject minority carriers which are collected at the specimen surface to form the EBIC image. At regions of enhanced minority carrier recombination the collected current is less than at defect free reginns. The images at these points therefore appear dark in the EBIC micrographs.

Finally, a multi-wire, real time Laue back refleetion-x-ray camera was used to determine twinning relationships in a variety of unthinned web material.

\section{Results}

\section{a. Web-Denarite Geometry}

The crystallographic orientation of the web and dendrites is shown schematically in Flgure 1. The dendrites must contaln ul least two planco before dendritic growth of a diamond lattice structure can occur(3) and, although it is not necessary, it is common to find some of these twin planes extending into the web. Both the $\mathrm{WI} 80-3.4 \mathrm{C}$ and $\mathrm{J} 135-3.6$ webs were Laue $\mathrm{x}$-rayed to determine the orientation relationship between opposite growth faces. Figure 2 shows an $x-r a y$ pnttiern taken with the beam striking the w180-3.4C web end-on. The (1II) twin relationship between opposite faces is evident showing that the web contains an odd number of twin planes. X-ray patterns from the $1335-3.6$ web determined that this sample contained an even number of twin planes. 
b. Defects at the Web Surface

Silicon web J135-3.6 was etched and optical micrographs taken at

various positions across the surface. These are shown in Figure 2 together with a sketch of the web surface indicating the areas corresponding to the micrographs. Figure $3(j)$ shows the relationship. between position and etch-pit density. The density is $\sim 5.10^{5}$ in the center of the web dropping to $\sim 5.10^{4}$ at the edges. It is generally accepted $(5,6)$ that most of the dislocations in silicon webs originate from highly strained regions in the dendrites, caused by liquid entrapment during growth. Such a region is shown in Figure 4, with the slip plane traces clearly visible. The maximum of about $5.10^{5}$ dislocations per $\mathrm{cm}^{2}$ observed in Figure $3(j)$ is due to thermal stresses generated during growth causing most of the dislocations to slip into the center of the web. If the stresses generated at a dislocation source are insufficient to promote slip, then these dislocations will remain at regions close to the dendrites. This explains the secondary maxima observed in Figure $3(j)$.

Figure 5 shows an EBIC micrograph of the web surface. The dark spots on the micrograph are caused by enhanced minority carrier recombination at dislocations inclined to the surface. The density. of electrically active defects observed in EBIC is the same as the etch-pit density observed in optical microscopy, suggesting that most, if not all, of the dislocations intersecting the web surface are electrically active. Solar cells with relatively high efficiencies have been fabricated from silicon web containing dislocation densities of the order of magnitude observed in the present study(7), indicating that either the electrical activity associated with these defects is not strong enough to significantly detract from cell performances or that the defect densities are at a tolerable level. 


\section{c. Electrical Activity at the Twin Planes}

The electrical activity of the twin boundaries was studied by beveling and polishing a specimen $(J 135-3.6)$ at a shallow angle, 1-2 ${ }^{\circ}$, to the growth surface. EBIC, see Figure 6, shows that a high density of dislocations is concentrated at the twin boundaries and that in this region strong minority carrier recombination processes occur. It is thought that the thermal stresses generated during growth also cause dislocations to slip to the interior of the web, where the twin planes act:as obstacles to further slip processes. Figure 7 is an optical micrograph of the same area as the EBIC image after the specimen had been etched to remove the Schottky diode and delineate the defect. As in the case of dislocations at the web surface, there is a close agreement between the density of dark EBIC features in Figure 6 and the optical etch pit density in Figure 7 . A direct correspondence between Figures 6 and 7 is not possible since the etching process removes several $u \mathrm{~m}$ of material from the surface. Both EBIC and optical micrographs show that two twin boundaries exist in the web, i.e., a microtwin, and that the dislocation density on one side of the microtwin is higher than on the other.

The fine line marked $A$ in Figure 6 is thought to be a small angle grain boundary; however, this has not yet been confirmed. The width and contrast features are not typical of surface scratches normally visible in EBIC micrographs and the contrast is probably due to the surface topology rather than to an associated electrical activity. Laue $x$-ray diffraction patterns from either side of the line failed to reveal any difference in orientation, indicating that, if the observed feature is indeed a low-angle boundary, the mismatch is $<1^{\circ}$. Similar features have been observed by the authors in other web specimens where they are visible on the surface faces. 
Figure 8 shows an EBIC micrograph taken from silicon web material WI80-3.4C, where the dislocations are almost surface parallel. The dislocations are centered around the active boundary shown in the middle of the micrograph. This is probably a twin boundary which has been forced out of the web during growth. The dislocations associated with it are also strongly electrically active.

\section{d. Defect Structure at the Twin Planes}

Previous investigators(8) identified the defect structure of twin boundaries in silicon web as complicated arrays of dislocations displaying <110> Burgers vectors, with the dislocation lines primarily directed along [2ī̄] and <110> directions. The present study has confirmed the existence of these dislocations and in addition has identified two sets of misfit dislocations. Figures 9 (a) - 9 (d) are HVEM micrographs of web J135-3.6 showing an hexagonal array of twin boundary dislocations imaged with different diffraction conditions. From contrast analysis the Burgers vectors of these dislocations were determined as being of the <2ll> type. Each set can be extinguished by using the appropriate \{220\} reflection. The observed Burgers vectors are in agreement with the prediction of the Bollman theory(9), that intrinsic dislocations in coherent twin boundaries are Shockley partials, $b=1 / 6<2177$. These dislocations take up a small twist component in the (11I) twinning plane. The twin relationship could be easily verified from selected area diffraction. The twist component estimated from $\theta=\frac{|b|}{d}$ is in the order of $0.01^{\circ}$ and is therefore too small to be detected by electron diffraction. The second set of misfit dislocations, Figure 10, consists of slightly curved dislocations running in the [2īi] direction. They are also contained in a (III) twin plane and take un a small tilt compunent. Most of these dislocations are $60^{\circ}$ partials having Burgers vectors $=1 / 6[\bar{I} 2 \bar{I}]$, all of the same sign of $\mathrm{b}$ as revealed by $+g,-g$, while others are perfect dislocations with Burgers vectors of $1 / 2\left[0 \overline{1} 1^{\circ}\right], 90^{\circ}$ 
and $\frac{1}{2}[1 \overline{10}], 30^{\circ}$. The diffraction vector in Figure $10(6)$ was chosen such that the partials were out of contrast. O'Hara and Schwuttke, using a large area scanning oscillator technique (SOT) identified the edge and $30^{\circ}$ perfect dislocations but did not resolve the $60^{\circ}$ partials. They also reported dislocations with Burgers vectors: [101] and [110] type lying in the [2īi] direction, although they also noted that such dislocations should not slip readily, as opposed to the ones reported above.

Figure 10 also shows straight dislocations running in the <ll0> directions, and part of an hexagonal network similar to that shown in Figure 9. Stereo micrographs show that these dislocations are not contained in the same (Iil) plane as the curved dislocations and must therefore belong to another twin plane, i.e., the other side of a microtwin. A strong interaction occurs between the dislocations running in the $\langle 110\rangle$ directions and the network of partials. The straight dislocations have <1I0> type Burgers vectors and are edge sessile. They form when dislocations slipping on elther (III), (III) or (III) planes intersect dislocations in the twin planes and form Lomer sessile dislocations. O'Hara and Schwlutt.kp proposed that the reaction for dislocations slipping on the (III) plane would be

$$
\begin{aligned}
& \frac{1}{2} a \cdot[01 \bar{I}]+\frac{1}{2} \text { a }[101]=\frac{1}{2} \text { a }[110] \\
& (1], 1) \text { plane (III) plane Lomer dislocation }
\end{aligned}
$$

The $\frac{1}{2}$ a [110]. Lomer dislocations, shown in Figure 10, lying along the [1]0] direction, can then dissociate into a Cottrell-Lomer lock by the following reaction. $\frac{1}{2}$ a $[110]=1 / 6$ a $[11 \overline{2}]+1 / 6$ a $[110]+1 / 6$ a $[112] \ldots$ It is likely that the dissociated partials are responsible for the interactions with the hexagonal network. 
Similar reactions occur for dislocations slipping on the (IIII) and (III) planes with the resulting Lomer dislocations having Burgers vectors $\frac{1}{2}$ a [011] and $\frac{1}{2} a[10 I]$ and line directions [OII] and [IOI] respectively. Whereas 0 ! Hara

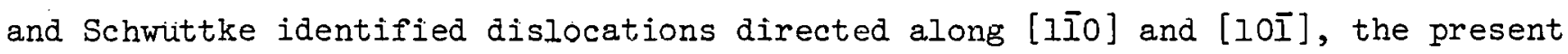
investigation has identified Lomer dislocations lying in all three of the <1IO> directions contained in the (1Il) twin plane.

In both the etch and EBIC studies"it was noted that the dislocation density was higher on one side of the microtwin than on the other. The HVEM studies have also shown that most of the dislocation reactions occur on one of the twin planes. It is likely that an inhomogeneous stress distribution is present in the material during growth, e.g. by buckling of the web.

\section{Conclusions}

The present study has shown that the defect structure in silicon web enhances minority-carrier recombination rates. The dislocations in the bulk of the material are electrically active. Empirically it is known that this activity does not significantly reduce the characteristics of solar cells. Since complete devices have not yet been investigated it is not known if the defect density is simply too low or if annealing reduces the electrical activity of dislocations(10). The surface parallel twin boundaries typical of this material are associated with a high density of dislocations. EBIC shows that these dislocations are sites of high minority carrier recombination and a reduction in solar cell efficiency is therefore expected when twin boundaries lie in the active region of a solar cell.

The defect structure of the twin planes has been investigated using HVEM, and soveral disluialion-types not previously reported in this material have been identified. 
5. Future Work

In order to fully understand the defect structure of silicon web and it.s effect on recombination processes, studies are underway to correlate the nature of the dislocations observed at the twin planes and their associated electrical activity. The occurrence of several types of dislocations and the relatively large spacing between them makes it an ideal candidate for direct EBIC and TEM correlations. In addition, an attempt. will be made to quantify the EBIC signals, thereby allowing a better comparison between the electrical activity of different types of defects. 
REFERENCES

I. S. N. Dermatis and J. W. Faust, "Silicon Sheets for the Manufacture of Semiconductor Devices", I.E.E.E. Trans. Communications Electronics 65, 194 (1963).

2. S. O!Hara and A. I. Bennett, J. Appl. Phÿs. 35, 686 (1964).

3. R. G. Seidensticker, J. Cryst. Growth 39, 17 (1977).

4. F. Secco d'Aragona, J. Electrochem. Soc. 119, 948 (1972).

5. S. O'Hara, J. Appl. Phys. 35, 409 (1964).

6. D. L. Barrett, E. H. Myers, D. R. Hamilton and A. I. Bennett, J. Electrochem. Soc. 118,952 (1971).

7. C. S. Duncan et al., "Silicon Web Process Development", JPL Technical Report, Contract No. NAS 954654, \#78/1, Westinghouse R\&D Center, 1978.

8. S. O'Hara and G. H. Schwittke, J. Appl. Phys. 36, 2475 (1965).

9. W. Bollman, Crystal Defects and Crystalline Interfaces, Springer Verlag, New York (1970).

10. H. Alexander, J. Physique Colioq. 40, C6-I (1979). 


\section{FIGURE CAPTIONS}

1. Schematic showing the crystallographic orientation of web-dendritic silicon.

2. Back reflection Laue $x$-ray pattern showing twin relationship in web material.

3. Optical micrographs of etch pits.

4. Optical micrograph of a dislocation sounce.

5. EBIC micrograph of a web surface.

6. ERIC micrograph of a bevelled surface showing electrical activity near twin boundaries.

7. Optical micrograph of same region as shown in Figure 6 .

8. EBIC micrograph of surface-parallel dislocations.

9. HVTEM micrographs of a dislocation network.g:diffraction vector.

10. HVIEM micrographs (see text for details). 


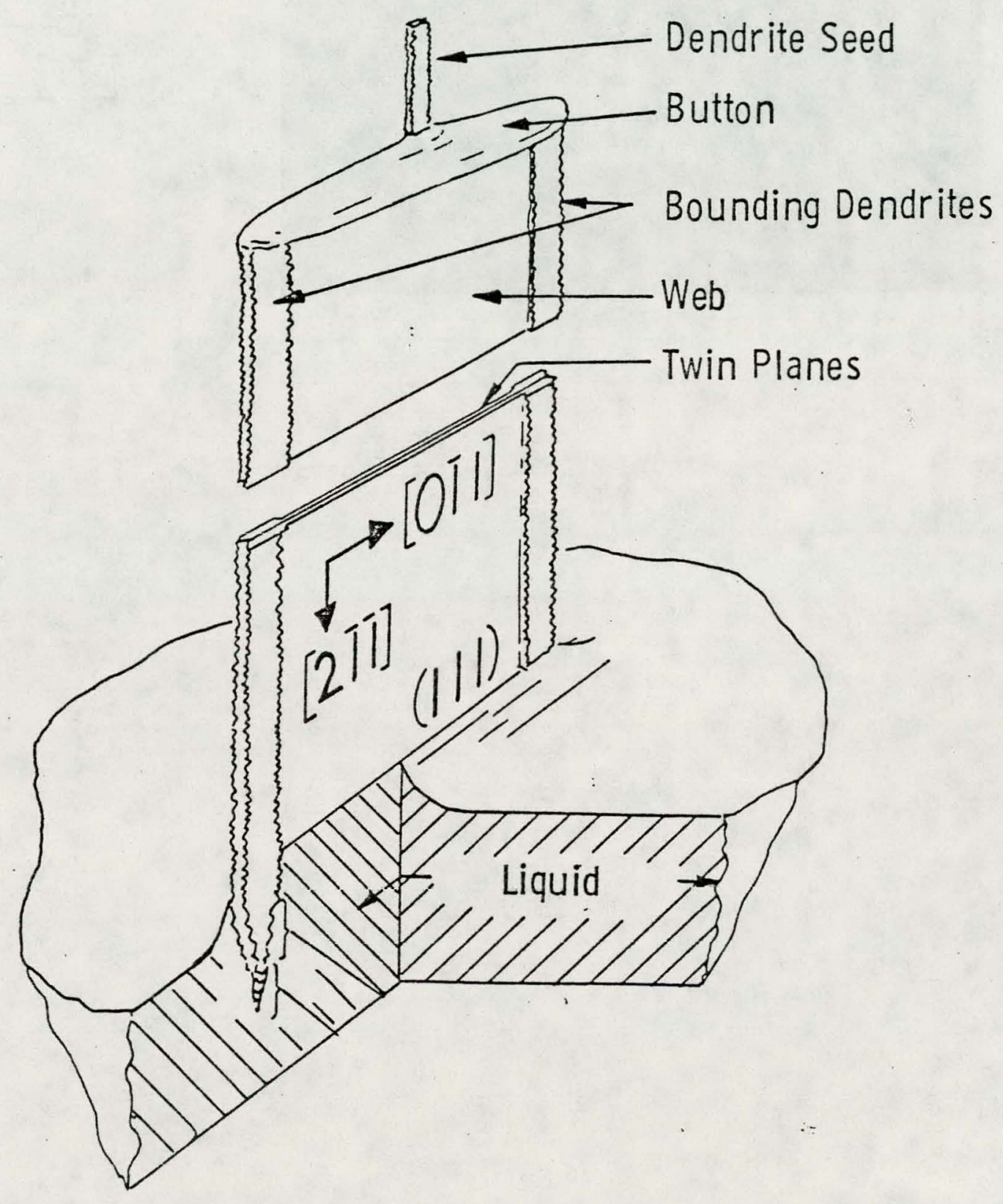

Figure 1 Schematic Section of Web Growth 

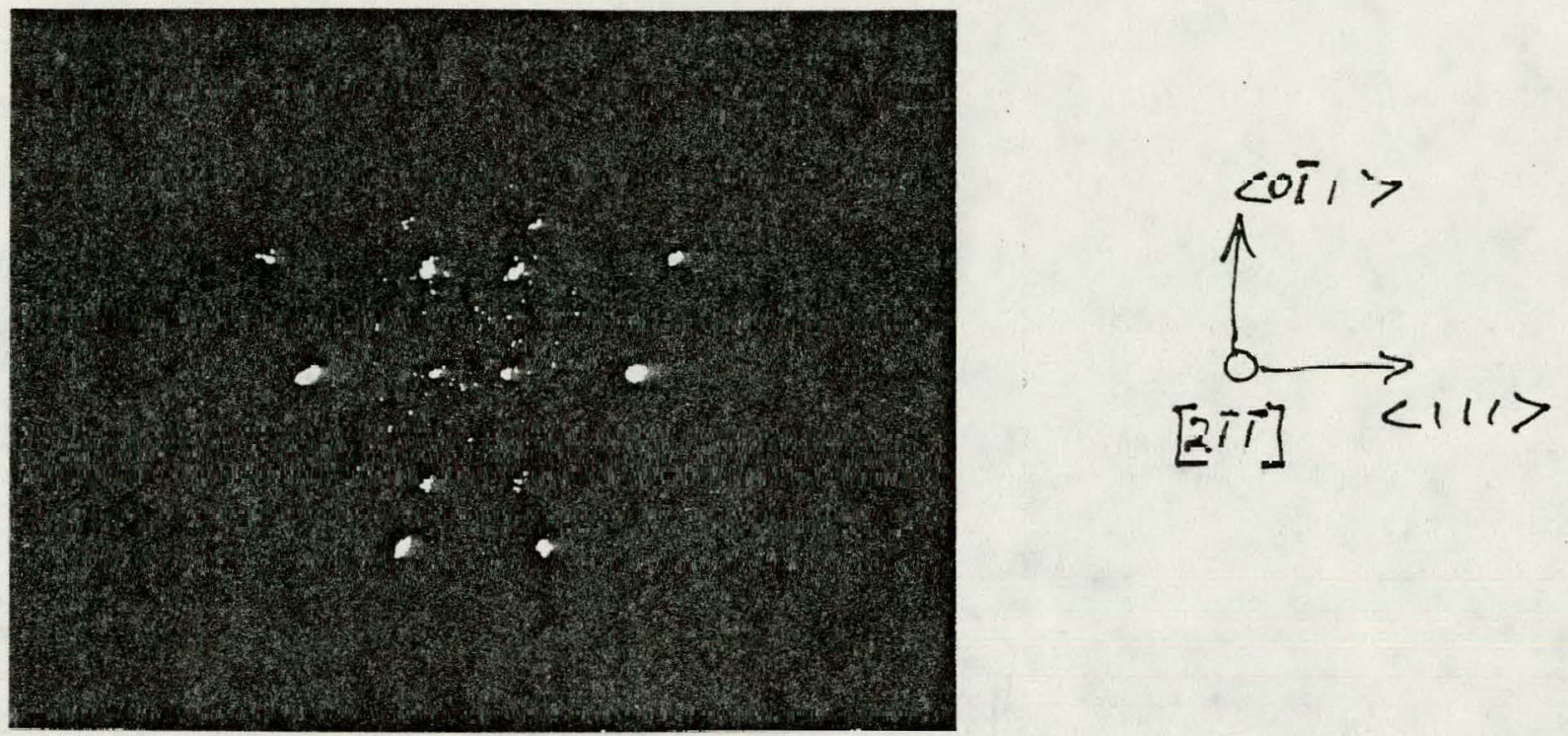

Figure 2 Laue $\mathrm{X}$-ray pattern. 


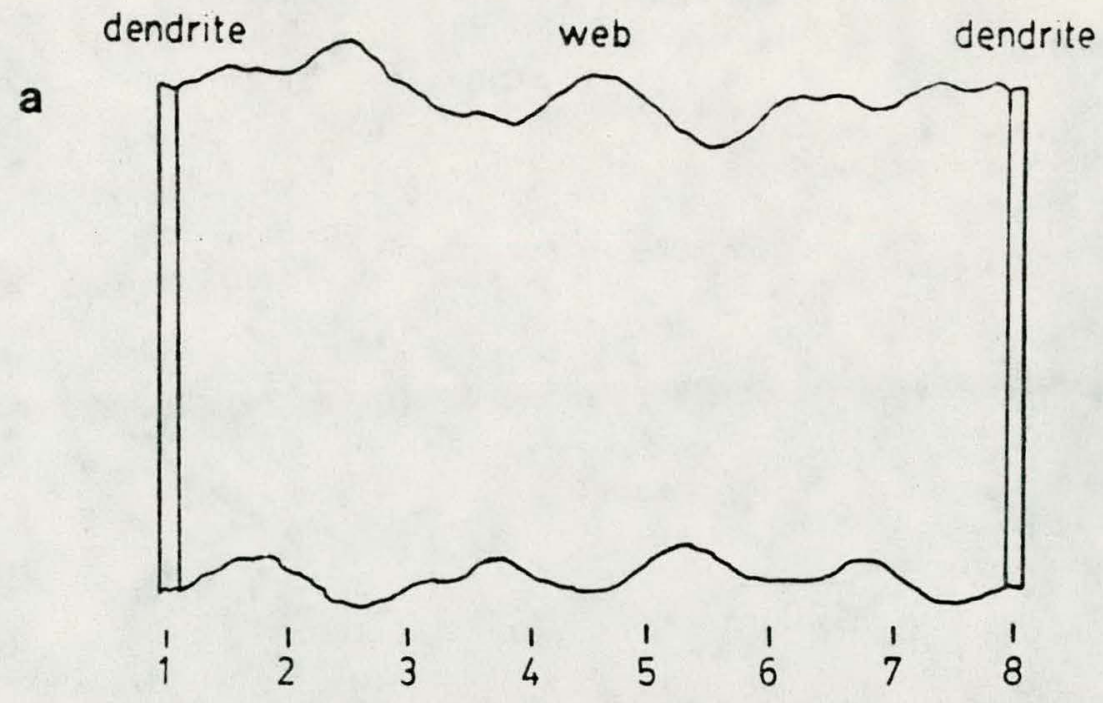

d
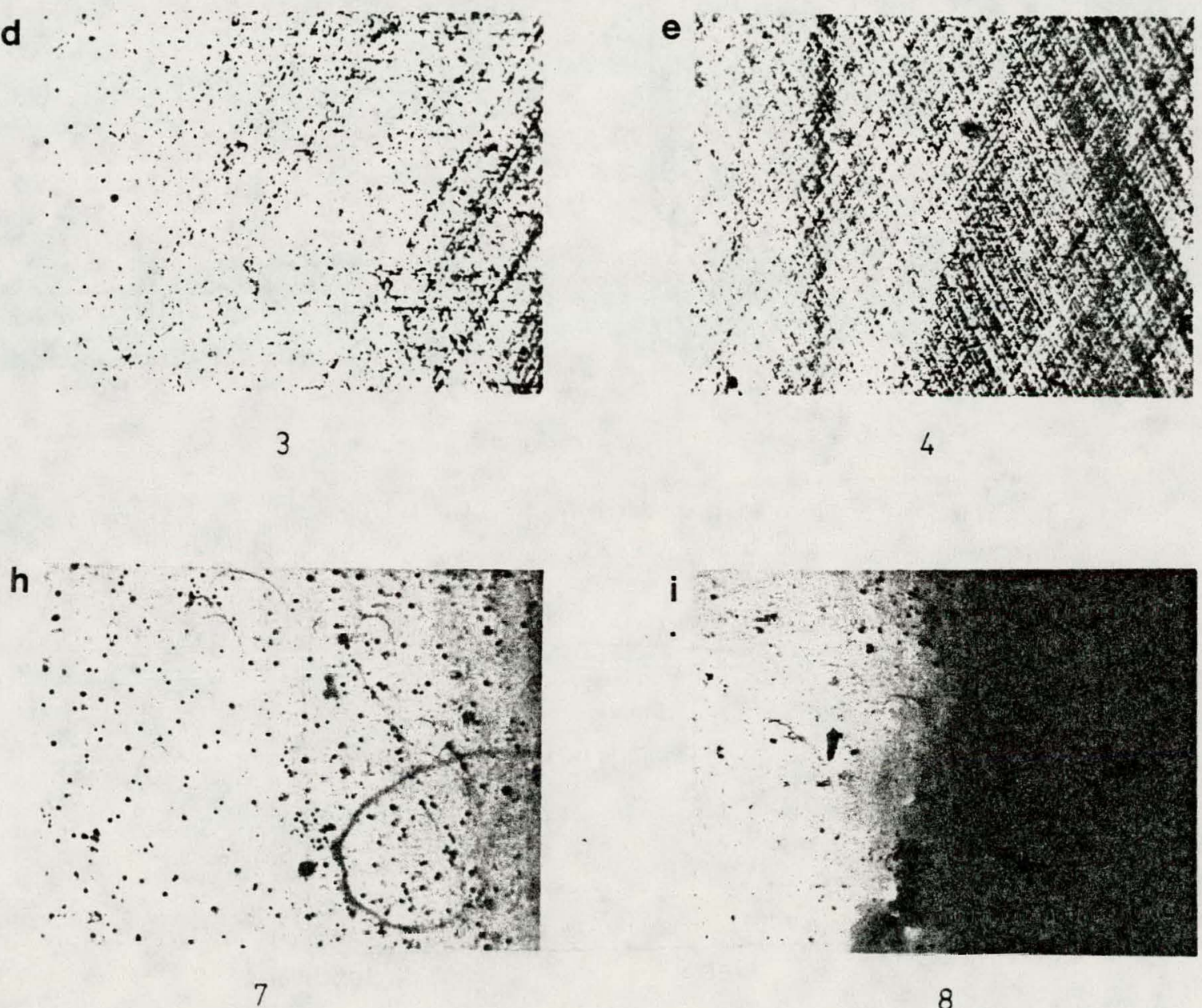

8

Dislocation etch pits in web-dendritic silicon

Fig. 3 
b
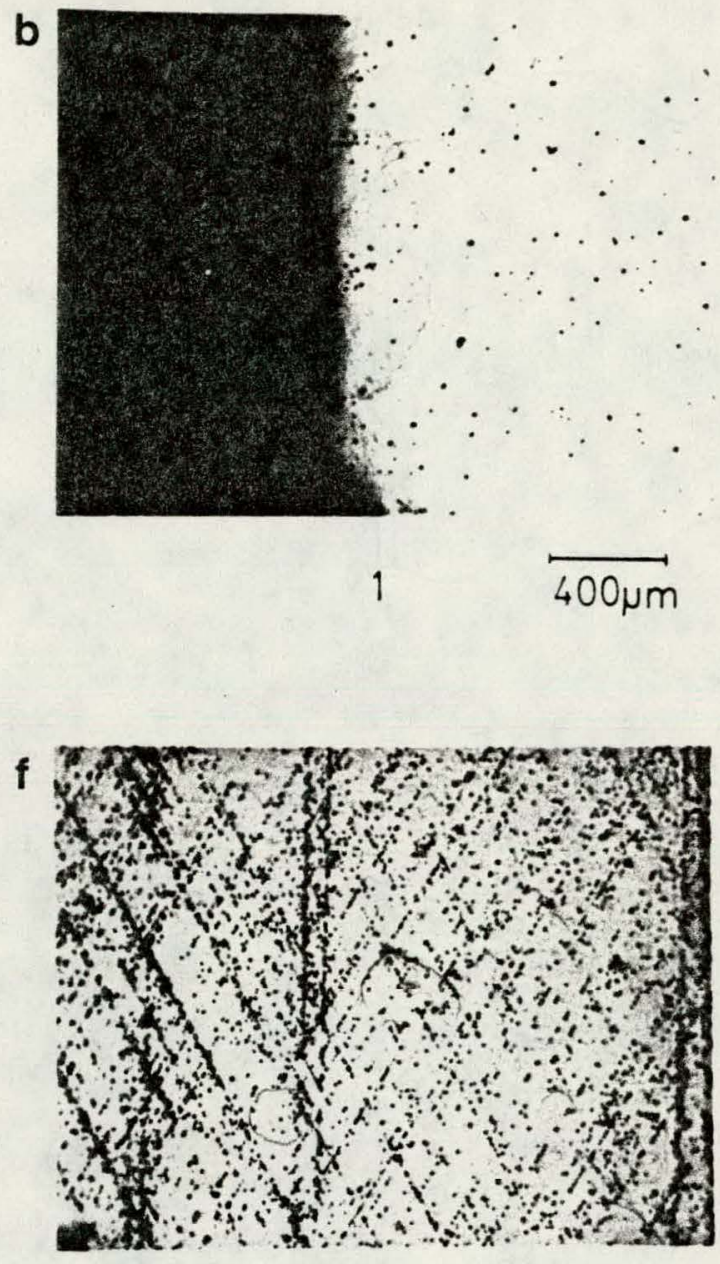

5
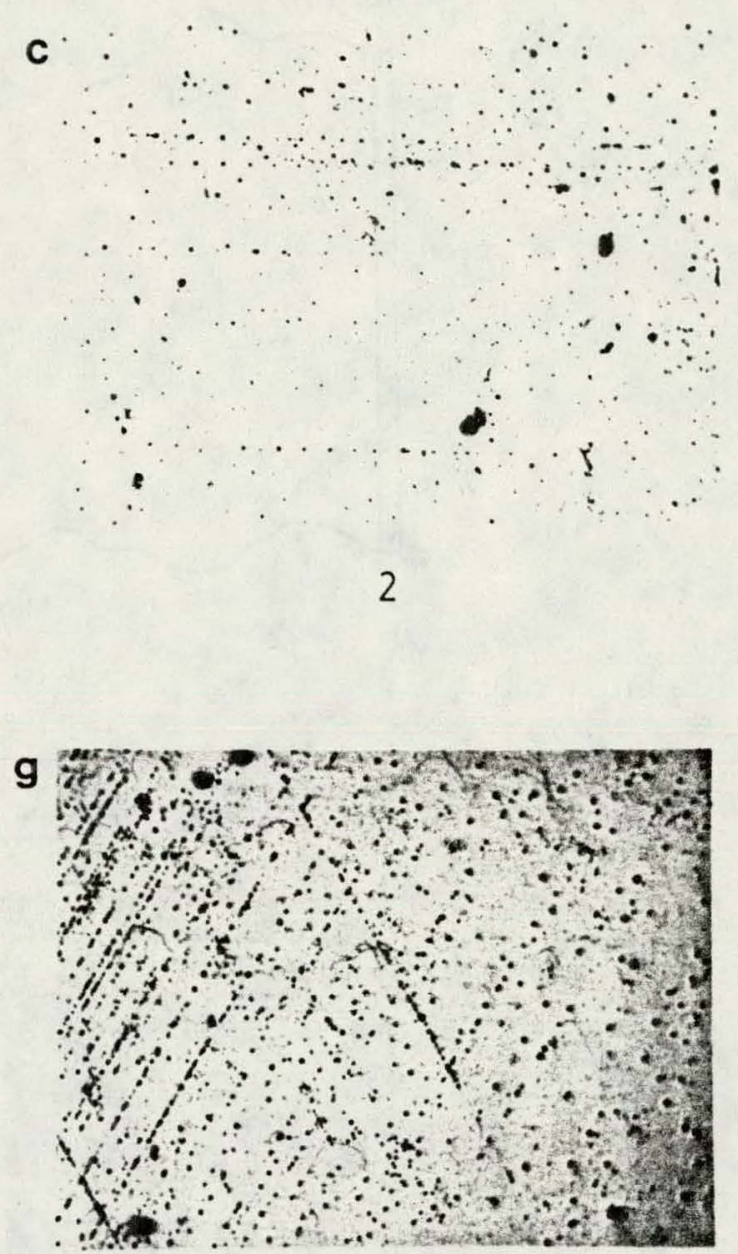

6

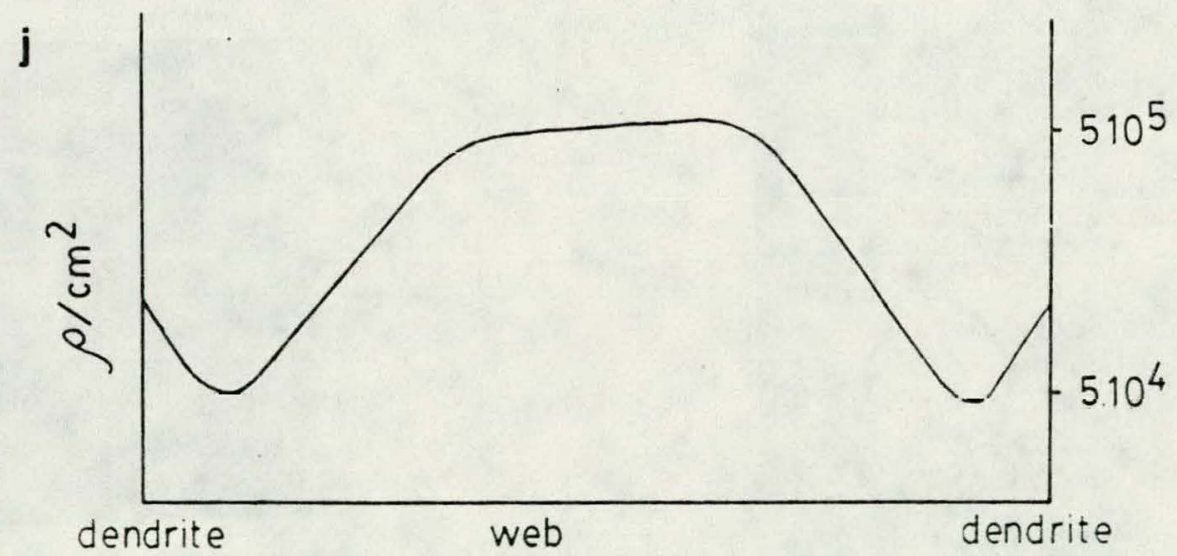

Dislocation etch pits in web-dendritic silicon

Fig. 3

B Cunningham / D G Ast 


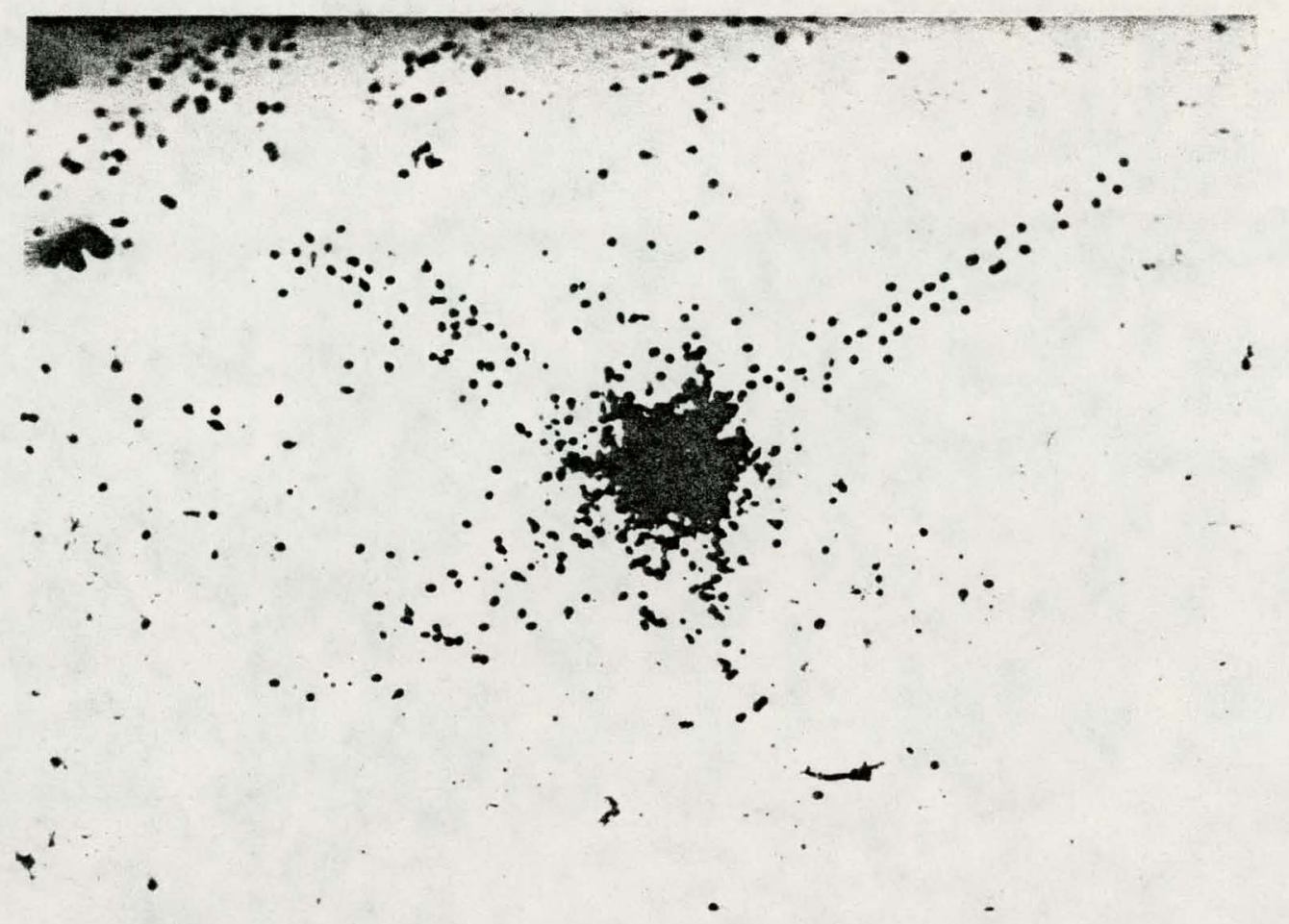

Fig. 4

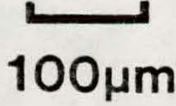


4

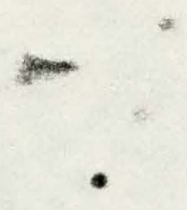

Fig. 5

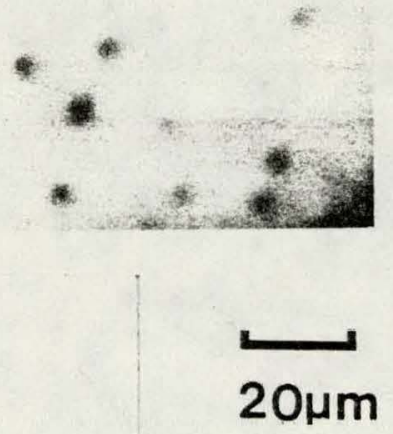




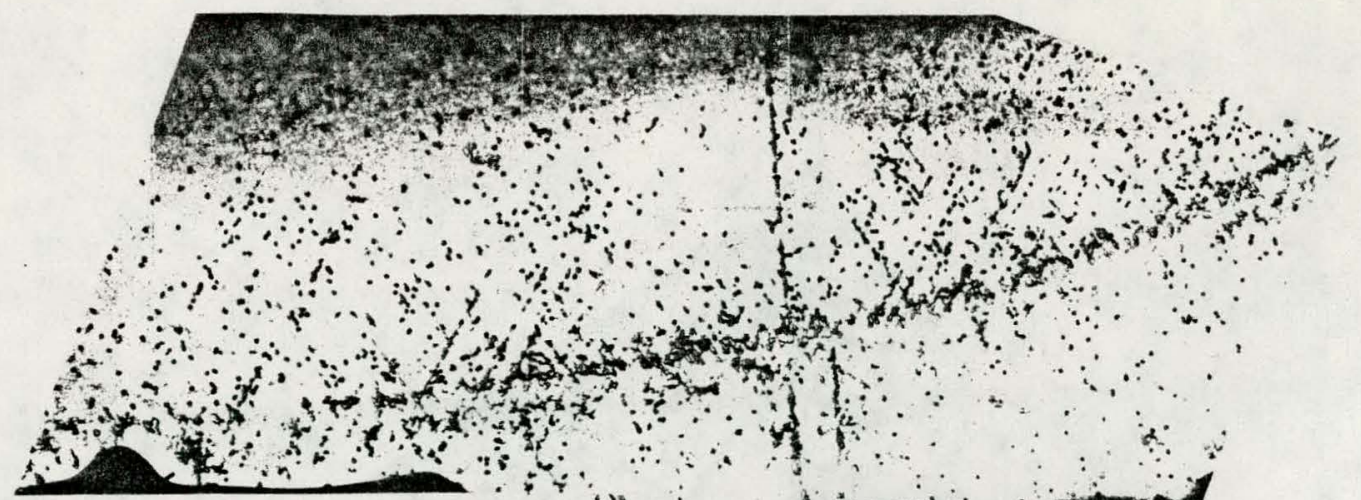

Fig. 7

$\overline{100 \mu \mathrm{m}}$

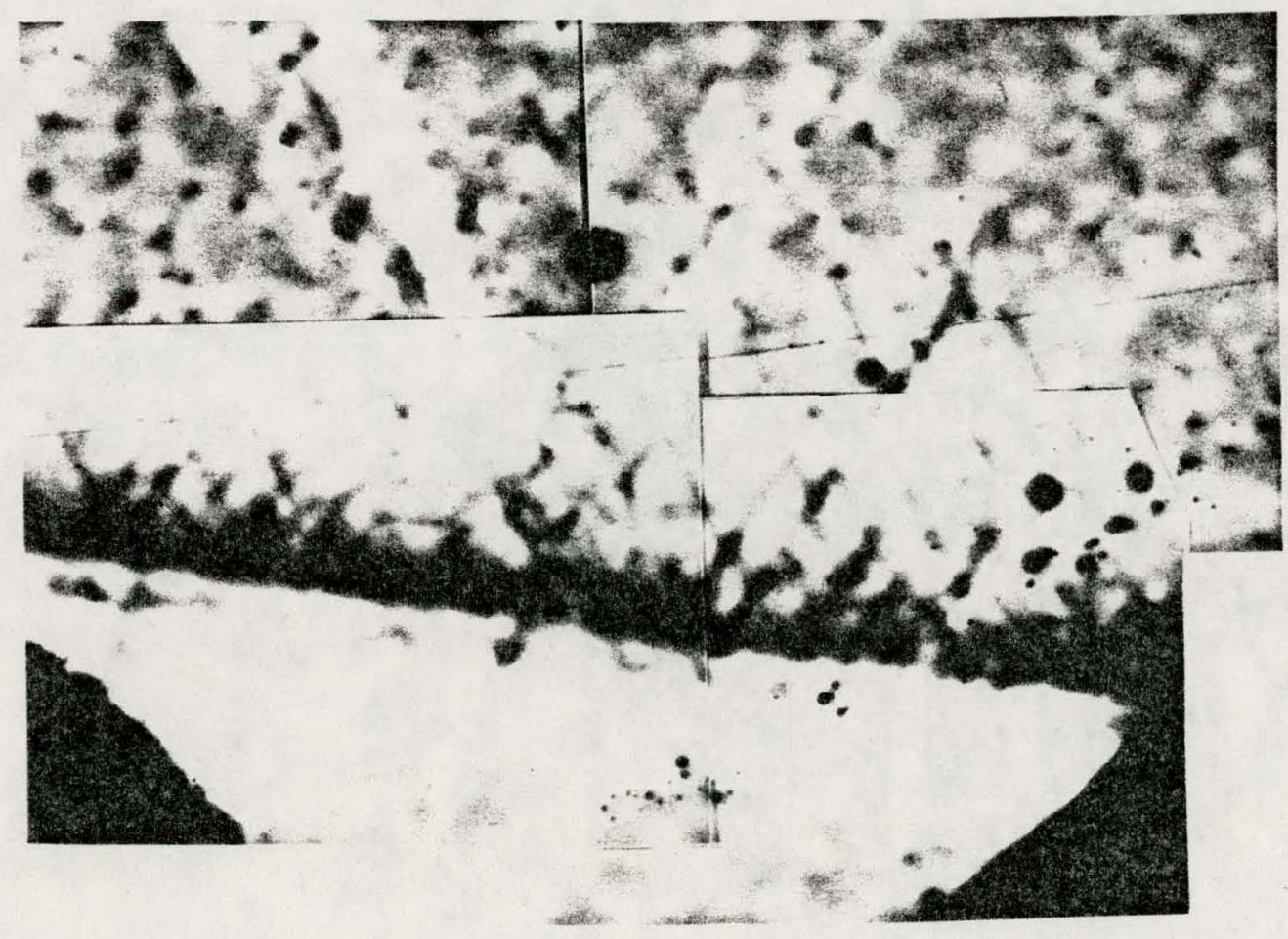

Fig. 6 


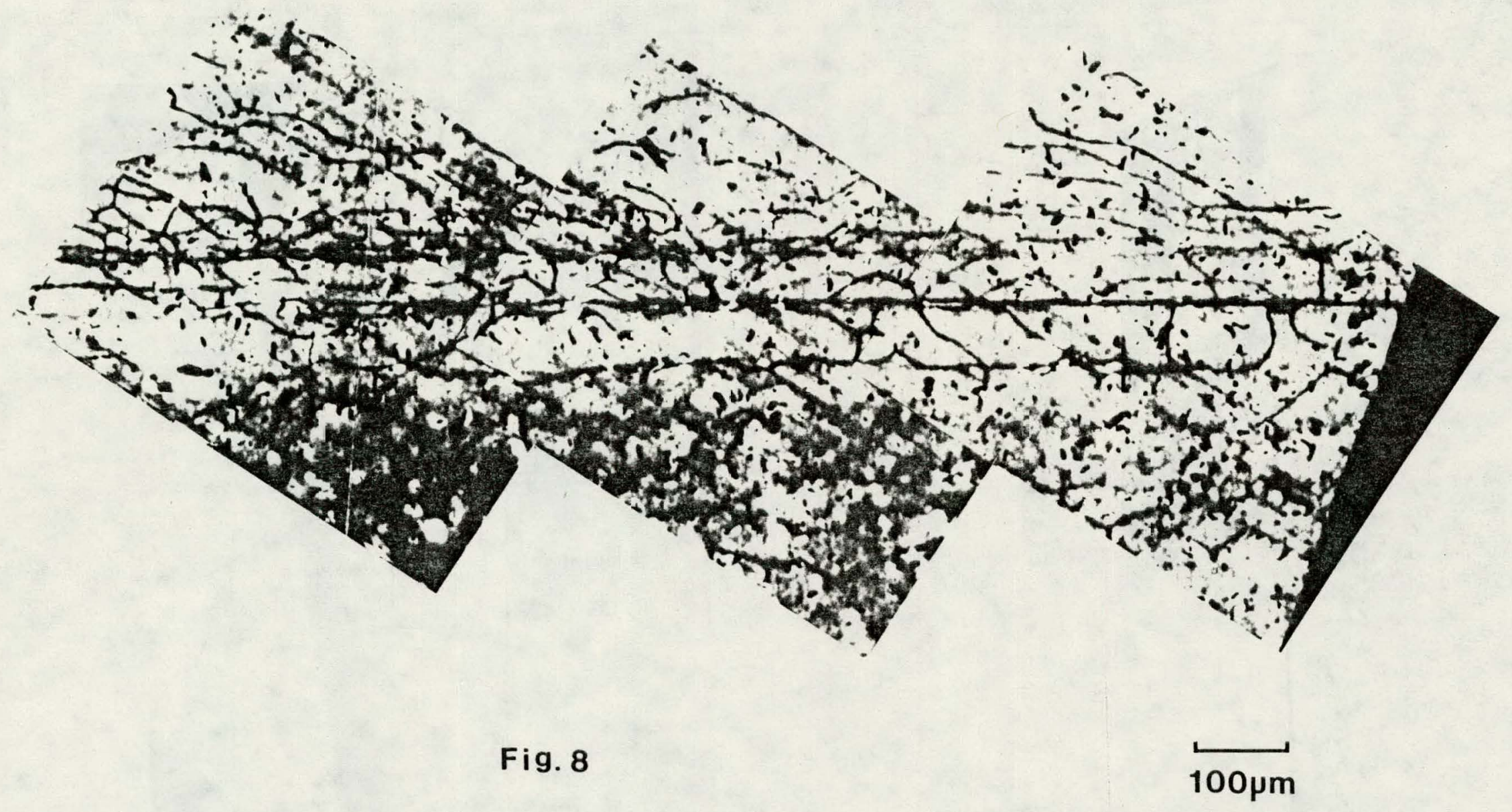


a

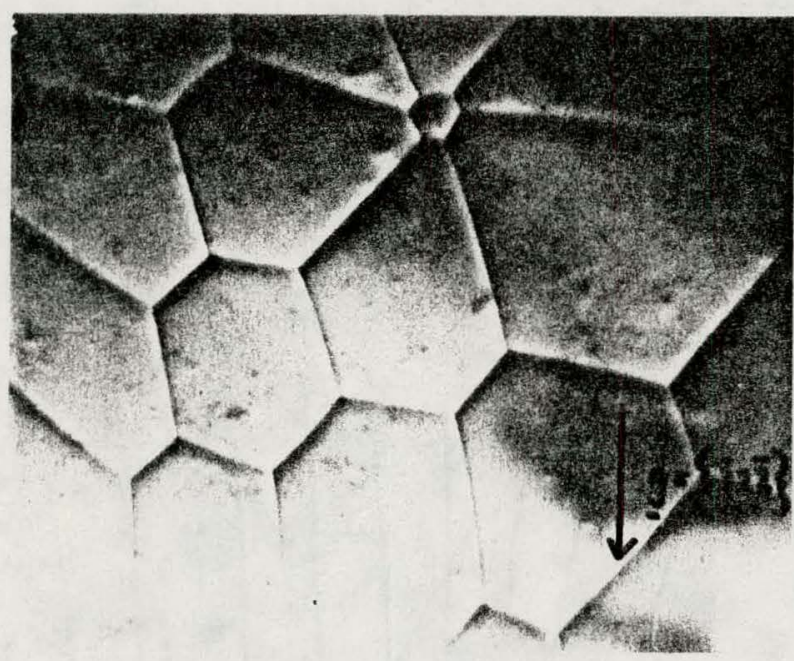

c

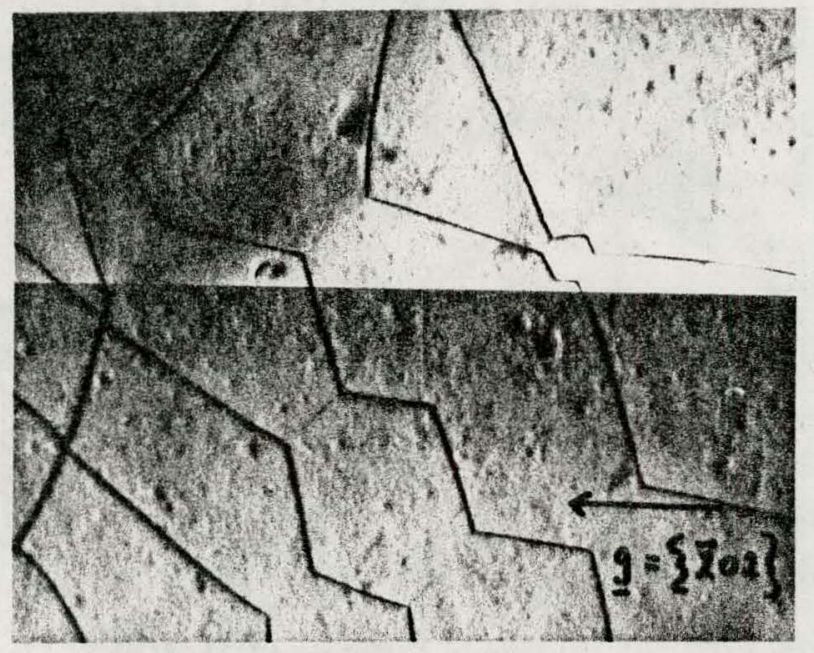

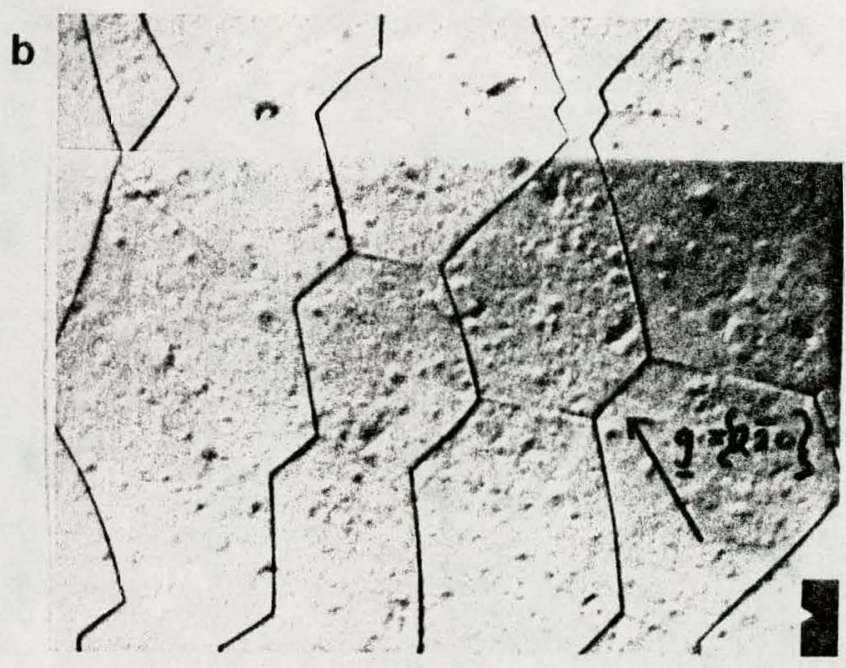

d

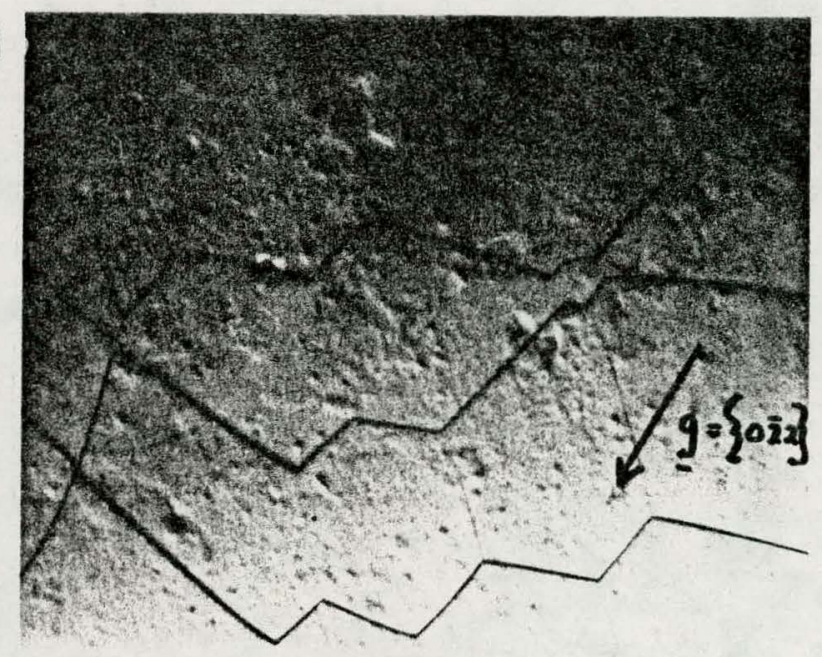

20um

Fig. 9 

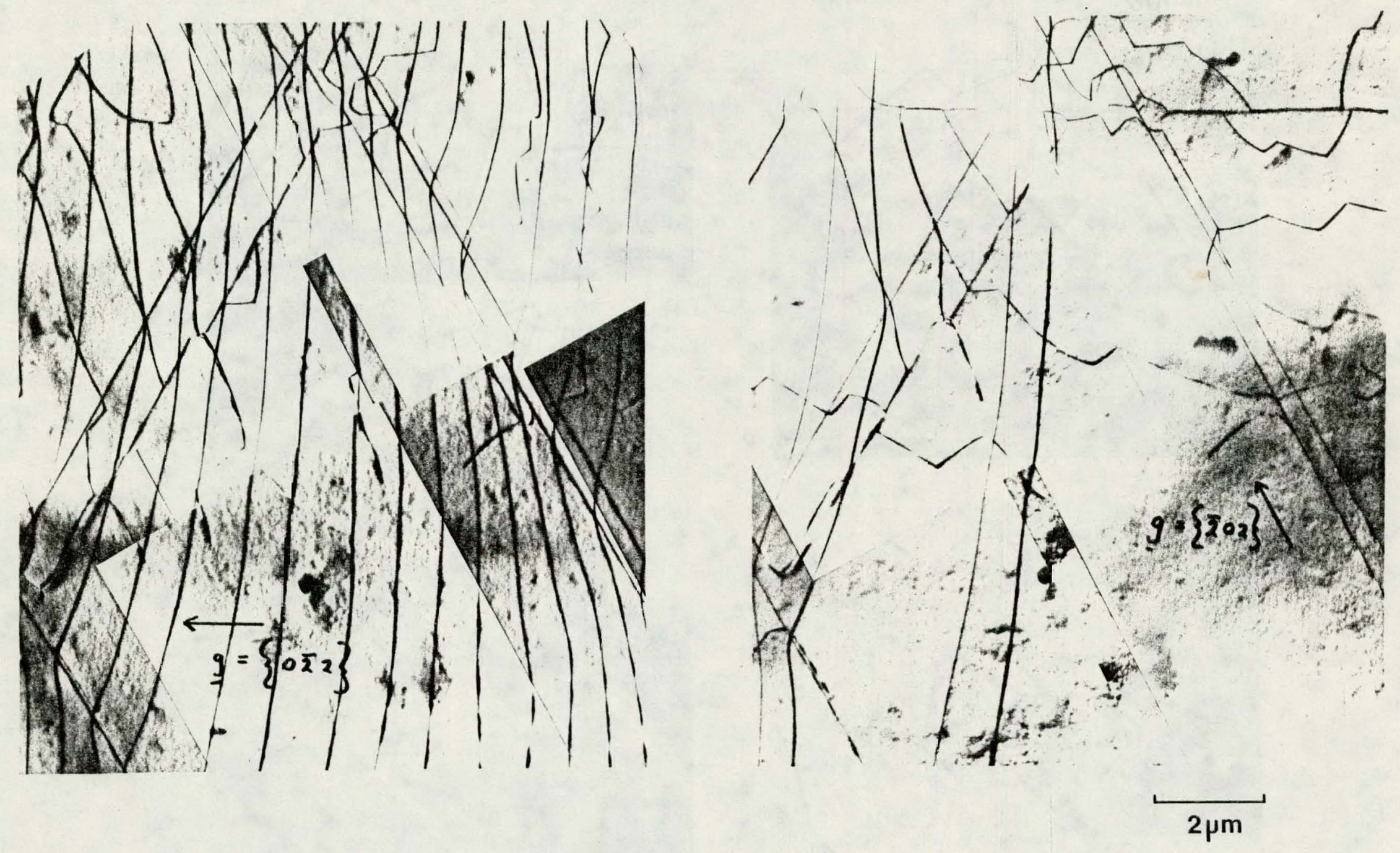

Fig. 10 